www.nature.com/cr

\title{
Self-eating with your fingers
}

\author{
Daniel J Klionsky ${ }^{1}$, James H Hurley² \\ ${ }^{1}$ Life Sciences Institute, and Department of Molecular, Cellular and Developmental Biology, University of Michigan, Ann Arbor, \\ MI 48109, USA; '2Laboratory of Molecular Biology, National Institute of Diabetes and Digestive and Kidney Diseases, National \\ Institutes of Health, Bethesda, MD 20892, USA \\ Cell Research (2012) 22:783-785. doi:10.1038/cr.2012.39; published online 13 March 2012
}

BECN1 plays a central role in determining cell fate, acting as a balance point in the decision to undergo autophagy or apoptosis. Three distinct structural domains allow BECN1 to interact with several other proteins. Recent crystallographic data provide insight into the nature of the coiledcoil and evolutionarily conserved domains.

Macroautophagy (hereafter autophagy) is a primarily degradative pathway that plays critical roles in cellular homeostasis [1]. The morphological and functional hallmark of autophagy is the double-membrane autophagosome, a sequestering compartment that is derived from the phagophore. One of the key components of the protein machinery that drives autophagy is $\mathrm{BECN} 1$, the product of the $B E C N 1$ gene and the homolog of yeast Vps30/Atg6. There are two phosphatidylinositol 3-kinase (PtdIns3K) complexes in yeast, both of which contain the PtdIns3K Vps34, the presumed regulatory subunit Vps15, and Vps30/Atg6 [2]. Complex I also includes Atg14, and is specific to autophagy, whereas this component is replaced in complex II by Vps38, which functions in endosomal trafficking.

Correspondence: Daniel J Klionsky $y^{\mathrm{a}}$, James H Hurley ${ }^{\mathrm{b}}$

aE-mail: klionsky@umich.edu

bE-mail: hurley@helix.nih.gov
BECN1 is part of at least three class III PtdIns3K complexes in mammals [3] that also include the PtdIns3K PIK3C3/ VPS34, PIK3R4/VPS15, and different combinations of ATG14/ATG14L/ BARKOR, UVRAG, AMBRA1 and/ or KIAA0226/RUBICON. The ATG14containing complex acts at an early stage of autophagosome formation, whereas UVRAG (which binds BECN1 at the same domain) directs the complex to act at a later stage of autophagosome maturation and also participates in endocytic trafficking. KIAA0226/ RUBICON localizes the PtdIns $3 \mathrm{~K}$ complex to late endosomes/lysosomes and acts to inhibit autophagy. The PtdIns3K complex plays a critical role in autophagy by synthesizing PtdIns3P, a phosphoinositide that recruits certain autophagy-related (ATG) proteins to the site of phagophore formation. BECN1 in particular has been the subject of much research, stemming in part from its identification as a tumor suppressor that is mutated in several human cancers. In addition to its autophagic function as a component of the PtdIns3K complex, BECN1 is connected to the apoptosis pathways via its ability to bind to antiapoptotic factors such as BCL2, BCL2L1/Bcl-XL and related proteins. A critical point regarding its function is that BECN1, along with AMBRA1 [4], may act as part of a balance point that controls the cellular response to stress, dictating whether the outcome is cytoprotective or results in cell death; for example, the BCL2-BECN1 complex inhibits autophagy and favors apoptosis. Accordingly, several regulatory molecules modulate autophagy through posttranslational modification of BECN1 or BCL2, including DAPK1 [5] and MAPK8/JNK1 [6]. Furthermore, BECN1 may also contribute to apoptosis following caspase cleavage and mitochondrial translocation [7], whereas the endoplasmic reticulumlocalized population of BECN1 is involved in promoting autophagy [8].

Despite the identification of over 30 Atg proteins in yeast, at least half of which are conserved in higher eukaryotes, relatively little structural information is available. Furthermore, almost all of the crystallography data has centered on the components of the ubiquitin-like protein conjugation systems [9]. The structure of the short BCL2-homology-3 (BH3, amino acids 105-125) domain bound to BCL2L1/ Bcl-XL was determined five years ago [10]. BECN1, however, interacts with a large number of other proteins [11, 12] and these interactions are mediated through additional domains. Now, two new studies bring a much larger portion of BECN1 into structural view. Li et al. [13] focused on the coiled-coil domain (CCD, amino acids 174-266 (the crystal structure is for residues 172-264, rat BECN1 numbering)), whereas Huang et al. [14] focused on an extended version 
of the evolutionarily conserved domain (ECD, originally defined as amino acids 267-337; actually human BECN1 residues 248-450). The extended ECD is a stable, folded minimal unit on its own, and so should now be considered the relevant structural and functional unit. Collectively, these structures span more than half of the molecule. Indeed, the crystallized fragments contain nearly 20 overlapping residues, making it possible to generate a composite model of nearly the entire ordered portion of BECN1 (Figure 1). The functional studies carried out in parallel with the crystallography have begun to assign functions to these various subregions of BECN1. The coiled coil seems to be a major locus for the assembly of BECN1 into the autophagy- and endosomespecific PtdIns3K complexes, whereas the ECD may have an unexpected role in membrane targeting.

The function of BECN1 in autophagy depends on its incorporation into the two major forms of the PIK3C3 complex, which contain either ATG14 or UVRAG. ATG14 and UVRAG both contain coiled coils responsible for their complex formation with the rest of PIK3C3. The coiled-coil regions of ATG14 and UVRAG target the BECN1 coiled coil, and together form a putative heterodimeric coil. The heterodimeric BECN1-ATG14 and BECN1-UVRAG coils have not been crystallized, but the homodimeric assembly of BECN1 with itself is now reported. This heterodimer is probably not functional in autophagy, since its assembly competes with ATG14 and UVRAG binding. Indeed, the full homodimeric assembly is sterically incompatible with the fold of the ECD, suggesting that when full-length BECN1 homodimerizes in solution, at least the first 6-7 turns of one coil would have to unravel. The homodimeric coiled coil contains charged residues and bulky amino acids at unfavorable positions that destabilize the structure, thus favoring assembly of BECN1ATG14 and BECN1-UVRAG heterodimers instead. The homodimeric structure serves for now as a surrogate for the functional heterodimers, as shown in Figure 1. The metastable nature of the BECN1 CCD thus appears to be critical for the correct localization and function of BECN1 in autophagy.

The ECD forms a compact structure consisting of four $\alpha$-helices, and three antiparallel $\beta$-sheets. It is now clear that the first helix of the ECD is actually the $\mathrm{C}$ terminus of the $\mathrm{CCD}$, and the remainder of the ECD has an elegant pseudo three-fold symmetry. Loop 4, which connects the second $\beta$-sheet to helix 3 , contains three aromatic amino acids - an aromatic finger-whose side-chains protrude away from the remainder of the protein. The ECD associates with membranes in vitro, and displays a preference for acidic lipids such as phosphadtidylserine, phosphatidylinositol, and cardiolipin,

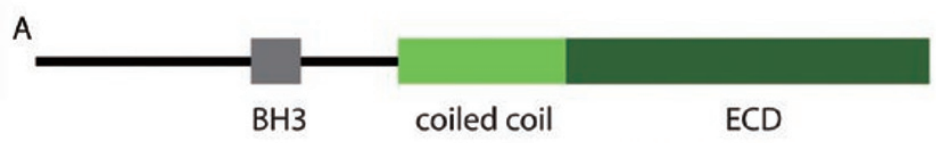

B ATG14/UVRAG

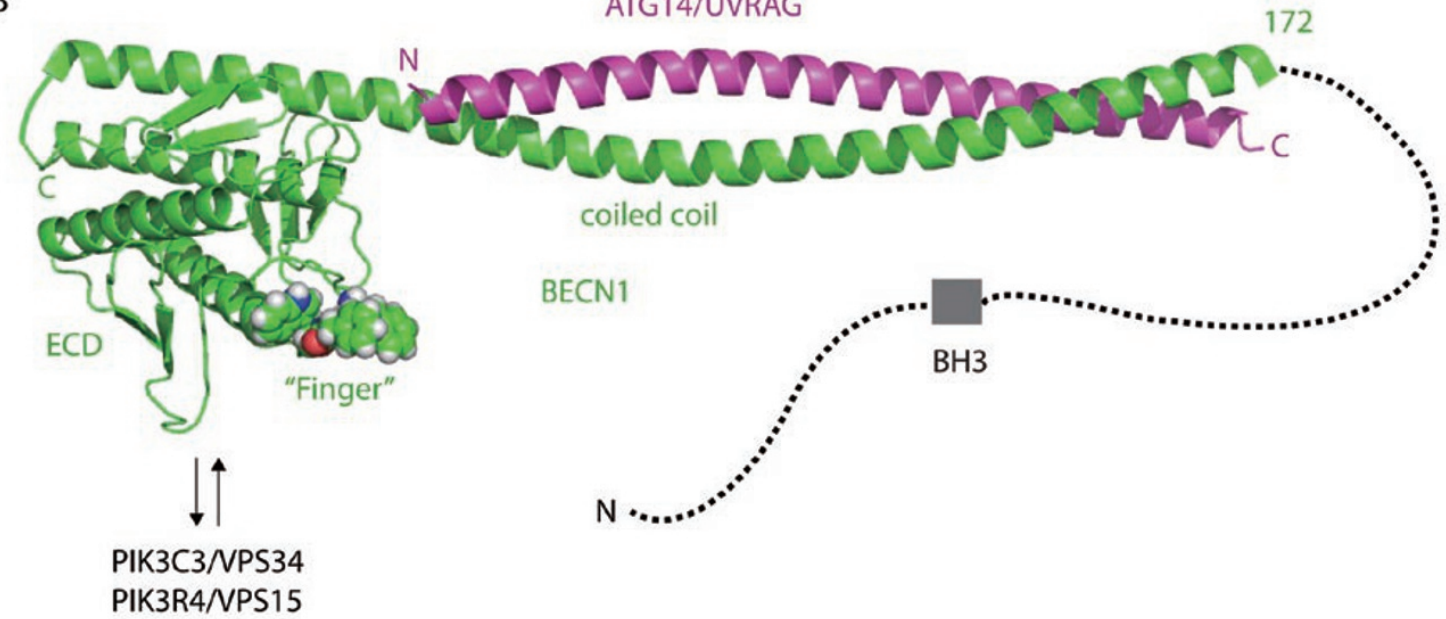

Figure 1 Composite model of the ordered portion of BECN1. (A) Schematic of the domain structure of BECN1. The black line denotes regions thought to be intrinsically disordered and flexible. (B) Structures of BECN1 ordered domains. The CCD and ECD structures were combined by superposition of the 19 overlapping residues in the two structures. In addition to the membrane binding role of the finger, the ECD was previously implicated in binding to the VPS34 and VPS15 subunits [20]. The second of the two coils of the CCD (magenta) is highlighted as a surrogate for the likely mode of binding of ATG14 or UVRAG. The second coil was truncated prior to residue 200 to avoid steric overlap with the ECD. The finger residues of the ECD are shown in a space-filling model. The black dotted line corresponds to the disordered regions in $\mathbf{A}$. 
suggesting that it may play a role in targeting BECN1 to the site of phagophore formation. In support of this hypothesis, mutation of the residues in the aromatic finger reduces membrane association in vitro and localization in vivo. The human BECN1 ECD joins at least two other recently identified domains, KA1 and MABP, that combine hydrophobic protrusions with basic surfaces in order to interact with acidic phospholipids $[15,16]$. Some of these other domains are thought to interact physiologically with the widely distributed monoanionic lipid phosphatidylserince. BECN1-ECD is proposed to interact with the bianionic mitochondrial lipid cardiolipin. It seems likely that the ECD finger will have additional functions beyond cardiolipin binding, given that the finger residues are not conserved in yeast and that PIK3C3 has activity at many cell membranes that are not enriched in cardiolipin.

One of the intriguing questions in the autophagy field concerns the mechanism that induces curvature of the phagophore membrane; there is no evidence for a protein coat similar to those that act in this manner throughout the secretory pathway. Electron microscopy data suggest that the ECD may cause liposomes to undergo deformation. The induction and sensing of membrane curvature is a complex topic [17] that with respect to the ECD has so far been addressed in a preliminary fashion, and will require further investigation. There are several other candidates for a membrane curvature-sensing element in the PIK3C3 interactome. For example, SH3GLB1 interacts with BECN1 via UVRAG; the former has a BAR domain that may be critical in membrane deformation for autophagy [18]. In addition, there are components that detect membrane curvature such as the BARKOR autophagosome targeting sequence (BATS) domain of ATG14 [19], which may be critical in the membrane recruitment of the PtdIns3K. Resolution of this question is just one facet of the world-class puzzles in molecular membrane biology: how is the phagophore nucleated, expanded and closed?

\section{Acknowledgments}

R Stanley is thanked for discussions. Research in the Hurley lab is supported by the Intramural Program of the NIH, NIDDK. DJK is supported by NIH grant GM53396.

\section{References}

1 Yang Z, Klionsky DJ. Eaten alive: a history of macroautophagy. Nat Cell Biol 2010; 12:814-822.

2 Kihara A, Noda T, Ishihara N, Ohsumi Y. Two distinct Vps34 phosphatidylinositol 3-kinase complexes function in autophagy and carboxypeptidase Y sorting in Saccharomyces cerevisiae. J Cell Biol 2001; 152:519-530.

3 Matsunaga K, Saitoh T, Tabata K, et al. Two Beclin 1-binding proteins, Atg14L and Rubicon, reciprocally regulate autophagy at different stages. Nat Cell Biol 2009; 11:385-396.

4 Strappazzon F, Vietri-Rudan M, Campello $\mathrm{S}$, et al. Mitochondrial BCL-2 inhibits AMBRA1-induced autophagy. EMBO J 2011; 30:11951208.

5 Zalckvar E, Berissi H, Mizrachy L, et al. DAP-kinase-mediated phosphorylation on the $\mathrm{BH} 3$ domain of beclin 1 promotes dissociation of beclin 1 from Bcl-XL and induction of autophagy. EMBO Rep 2009; 10:285-292.

6 Wei Y, Pattingre S, Sinha S, Bassik M, Levine B. JNK1-mediated phosphorylation of $\mathrm{Bcl}-2$ regulates starvationinduced autophagy. Mol Cell 2008; 30:678-688.

7 Wirawan E, Vande Walle L, Kersse K, et al. Caspase-mediated cleavage of Beclin-1 inactivates Beclin-1-induced autophagy and enhances apoptosis by promoting the release of proapoptotic factors from mitochondria. Cell Death Dis 2010; 1:e18.

8 Maiuri MC, Criollo A, Tasdemir E, et al. $\mathrm{BH} 3$-only proteins and $\mathrm{BH} 3$ mimetics induce autophagy by competitively disrupting the interaction between Beclin 1 and $\mathrm{Bcl}-2 / \mathrm{Bcl}-\mathrm{X}(\mathrm{L})$. Autophagy 2007; 3:374-376.
9 Geng J, Klionsky DJ. The Atg8 and Atg12 ubiquitin-like conjugation systems in macroautophagy. EMBO Rep 2008; 9:859-864.

10 Oberstein A, Jeffrey PD, Shi Y. Crystal structure of the Bcl-XL-Beclin 1 peptide complex: Beclin 1 is a novel BH3-only protein. J Biol Chem 2007; 282:13123-13132.

11 Criollo A, Niso-Santano M, Malik SA, et al. Inhibition of autophagy by TAB2 and TAB3. EMBO J 2011; 30:49084920.

12 Behrends C, Sowa ME, Gygi SP, Harper JW. Network organization of the human autophagy system. Nature 2010; 466:68-76.

13 Li X, He L, Che KH, et al. Imperfect interface of Beclin1 coiled-coil domain regulates homodimer and heterodimer formation with Atg14L and UVRAG. Nat Commun 2012; 3:662.

14 Huang W, Choi W, Hu W, et al. Crystal structure and biochemical analyses reveal Beclin 1 as a novel membrane binding protein. Cell Res 2012; 22:473489.

15 Moravcevic K, Mendrola JM, Schmitz $\mathrm{KR}$, et al. Kinase associated-1 domains drive MARK/PAR1 kinases to membrane targets by binding acidic phospholipids. Cell 2010; 143:966-977.

16 Boura E, Hurley JH. Structural basis for membrane targeting by the MVB12-associated $\beta$-prism domain of the human ESCRT-I MVB12 subunit. Proc Natl Acad Sci USA 2012; 109:1901-1906.

17 McMahon HT, Kozlov MM, Martens S. Membrane curvature in synaptic vesicle fusion and beyond. Cell 2010; 140:601-605.

18 Takahashi Y, Coppola D, Matsushita $\mathrm{N}$, et al. Bif-1 interacts with Beclin 1 through UVRAG and regulates autophagy and tumorigenesis. Nat Cell Biol 2007; 9:1142-1151.

19 Fan W, Nassiri A, Zhong Q. Autophagosome targeting and membrane curvature sensing by Barkor/Atg14(L). Proc Natl Acad Sci USA 2011; 108:77697774.

20 Furuya N, Yu J, Byfield M, et al. The evolutionarily conserved domain of Beclin 1 is required for $\mathrm{Vps} 34$ binding, autophagy and tumor suppressor function. Autophagy 2005; 1:46-52. 